\title{
Epidemiological characterization of imported recurrent Plasmodium vivax and Plasmodium ovale in China, 2013-2020
}

Yanwen Cui ${ }^{1,2,3,4 \dagger}$, Li Zhang ${ }^{1,2,3,4 \dagger}$, Zhigui Xia ${ }^{1,2,3,4}$, Hejun Zhou ${ }^{1,2,3,4}$ and Fang Huang 1,2,3,4*

\begin{abstract}
Background: China has reached important milestones in the elimination of malaria. However, the numbers of imported recurrent cases of Plasmodium vivax and P. ovale are gradually increasing, which increases the risk of malaria re-establishment in locations where Anopheles mosquitoes exist. The aim of this study is to characterize the epidemiological profiles of imported recurrent $P$. vivax and $P$. ovale cases, quantifying the recurrence burden and guiding the development of appropriate public health intervention strategies.

Methods: Individual-level data of imported recurrent P. vivax and P. ovale cases were collected from 2013 to 2020 in China via the Parasitic Diseases Information Reporting Management System. Demographic characteristics, temporal and spatial distributions, and the interval from previous infection to recurrence were analyzed by SAS, ArcGIS and GraphPad Prism software, respectively, to explore the epidemiological profiles of imported recurrent cases.

Results: A total of 307 imported recurrent cases, including 179 P. vivax and 128 P. ovale cases, were recorded. The majority of cases occurred in males (P. vivax 91.1\%, P. ovale 93.8\%) and migrant workers (P. vivax 43.2\%, P. ovale 44.7\%). Individuals aged 30-39 years had the highest $P$. vivax and $P$. ovale recurrent infection rates, respectively. The number of imported recurrent cases of infection by these two malaria species increased from 2013 to 2018, and $P$. vivax infection showed well-defined seasonality, with two peaks in February and June, respectively. More than $90 \%$ of patients with recurrent cases did not receive radical treatment for previous infection. Most imported recurrent $P$. vivax cases were reported in Yunnan Province and were imported from Myanmar, Ethiopia, and Pakistan, while most recurrent P. ovale cases were reported in southern China and primarily imported from Cameroon, Ghana, and Nigeria. The intervals from previous malaria infection to recurrence among different continents were significantly different $(P=0.0016)$ for $P$. vivax malaria but not for $P$. ovale malaria $(P=0.2373)$.
\end{abstract}

Conclusions: The large number of imported recurrent cases has been a major challenge in the prevention of malaria re-establishment in China. This study provides evidence to guide the development of appropriate public health intervention strategies for imported recurrent $P$. vivax and $P$. ovale cases.

Keywords: Recurrence, Imported malaria, Plasmodium vivax, Plasmodium ovale, China

*Correspondence: huangfang@nipd.chinacdc.cn

${ }^{\dagger} Y a n w e n$ Cui and Li Zhang contributed equally to this work.

${ }^{1}$ National Institute of Parasitic Diseases, Chinese Center for Disease

Control and Prevention (Chinese Center for Tropical Diseases Research), Shanghai 200025, China

Full list of author information is available at the end of the article

\section{Background}

Malaria imposes a heavy disease burden globally. In 2019, an estimated 229 million malaria cases and 409000 malaria-related deaths were reported in 87 malaria endemic countries [1]. The majority of malariarelated morbidity and mortality is caused by two of the five Plasmodium species that naturally infect humans: 
P. falciparum and P. vivax [2]. P. vivax is the most geographically widespread cause of human malaria and the heaviest burden of $P$. vivax infection occurs throughout Southeast Asia, South America, and sub-Saharan Africa [3], where an estimated 2.5 billion people are at risk of infection [4].

In recent years, many countries have gradually achieved malaria pre-elimination or elimination through the upscaling of well-planned multipronged intervention strategies [5]. $P$. vivax and $P$. ovale are often the last parasites to be eliminated in malariaelimination settings. The possibility of recurrent $P$. vivax or $P$. ovale infection in patients is a barrier to successful malaria treatment and effective control [6]. Recurrent $P$. vivax and $P$. ovale cases can be hypnozoite-derived (relapse) or caused by blood-stage treatment failure (recrudescence) or newly acquired infection (reinfection) [7]. Primaquine (PQ), the only widely available antimalarial drug with hypnozoitocidal activity for radical treatment to prevent relapse, can cause life-threatening haemolysis in humans with glucose-6-phosphate dehydrogenase (G6PD) deficiency [8]. Until now, distinguishing among these three different causes of recurrent infection has remained a challenge. Therefore, understanding the recurrence burden and epidemiological characteristics of recurrent cases will be significant in achieving malaria elimination, especially $P$. vivax.

In China, four human Plasmodium species (P. vivax, P. falciparum, $P$. ovale and $P$. malariae) were endemic, and $P$. vivax was the most widely distributed. From 2001 to 2006, a local P. vivax outbreak in the HuangHuai Plain in Central China occurred [9]. Since 2010, when the National Malaria Elimination Action Plan was launched, China has reached several malaria elimination milestones [10-12]. However, with increasing globalization, large numbers of individuals migrate from malaria-endemic countries or regions to China. Recurrent cases of imported $P$. vivax malaria have been reported and $P$. ovale has become the second most common of imported malaria in China, after P. falciparum [12]. Imported recurrent cases may increase the risks of re-establishment in malaria-free localities where Anopheles mosquitoes still exist. Thus, data to quantify the recurrence burden and guide the development of appropriate public health intervention strategies are urgently required $[13,14]$.

This study aimed to characterize the epidemiological profiles of imported recurrent $P$. vivax and $P$. ovale cases, providing evidence-based data that could support the adjustment of appropriate strategies during the post-elimination phase in China.

\section{Methods}

\section{Data collection}

Malaria data including Plasmodium species, case classification, demographic information (age, sex and occupation), radical treatment, source of malaria, date of onset, and date of previous illness from 31 provinciallevel administrative divisions collected via the Parasitic Diseases Information Reporting Management System (PDIRMS) from January 1, 2013, to December 31, 2020, were reviewed. Data from Hong Kong, Macao, and Taiwan were not included in the study.

According to malaria diagnostic criteria in China [15], $P$. vivax and $P$. ovale cases were confirmed by microscopy, polymerase chain reaction (PCR), or rapid diagnostic tests (RDTs) in the laboratory. Imported malaria was defined as (i) malaria whose origin could be traced to a transmission area outside of China; (ii) a diagnosis of malaria; (iii) travel to a malaria-endemic area outside of China during the malaria transmission season; and (iv) a malaria onset time less than 1 month after returning to China during the local transmission season. In addition, according to the definition of imported cases, if the cases had a travel history to countries where malaria transmission occurs and the onset time for malaria was less than 1 month after returning to China, this country was defined as the country of origin for imported malaria cases. A recurrent case was defined as the recurrence of asexual $P$. vivax or $P$. ovale parasitaemia that was mainly caused by relapse, recrudescence or reinfection. Relapse was defined as hypnozoite recurrence. Recrudescence was defined as recurrence of asexual parasitaemia with parasites of the same genotype(s) that caused the original illness, due to incomplete clearance of asexual parasites after antimalaria treatment. Reinfection was defined as a new infection that followed the primary infection. Diagnosed $P$. vivax and $P$. ovale cases meeting any of the recurrence definitions were included in the study.

\section{Data analysis}

The epidemiological characteristics of imported recurrent cases of $P$. vivax and $P$. ovale were analysed. Temporal distributions were analysed by year and month using the software Microsoft Excel 2010 (Microsoft, Redmond, WA, USA). Statistical analysis was performed by the statistical software SAS 9.4 (SAS Institute Inc, NC, USA). Fisher's exact test was used to evaluate differences in demographic characteristics among the different subgroups. Interval data were visualized using the "violin plot" package in GraphPad Prism 8.4.3 (GraphPad Software, LLC., San Diego, CA, USA) to show the distribution and probability density. The Kruskal-Wallis test was used to analyse the interval distribution among different 
continents. Maps were created using ArcGIS 10.1 (Environmental Systems Research Institute, Inc., Redlands, CA, USA). A $P$ value $<0.05$ was considered to indicate a statistically significant difference.

\section{Results}

Demographic characteristics of imported recurrent cases

A total of 4679 imported $P$. vivax and 2202 imported $P$. ovale cases were recorded in the PDIRMS between January 1, 2013 and December 31, 2020, of which 307 were recurrent cases, including 179 recurrent $P$. vivax and 128 recurrent $P$. ovale cases. Recurrent cases accounted for $3.8 \%$ and $5.8 \%$ of the total imported cases of $P$. vivax and $P$. ovale, respectively. The majority of recurrent cases were reported in males ( $P$. vivax $91.1 \%$, P. ovale 93.8\%). The highest numbers of recurrent $P$. vivax and $P$. ovale cases were observed in the same age group, which were patients aged 30-39 years. The proportions of patients infected with these two Plasmodium species in the different age groups were significantly different $(P<0.001)$. In addition, most recurrent cases occurred in workers which included migrant workers ( $P$. vivax 43.2\%, $P$. ovale $44.7 \%)$, construction workers (P. vivax 38.2\%, $P$. ovale $21.3 \%$ ) and other workers (P. vivax $19.7 \%$, P. ovale $34.0 \%)$. The proportion of recurrent $P$. ovale infection was significantly different with that of $P$. vivax infection in occupation $(P<0.05)$. More than $90 \%$ of the patients with imported recurrent cases did not receive radical treatment for the previous infection (P. vivax 91.3\%, P. ovale 91.7\%) (Table 1).

\section{Temporal distributions of imported recurrent cases}

The number of imported $P$. vivax cases showed a decreasing trend from 2013 to 2020, while the proportion of imported recurrent $P$. vivax cases increased after 2016, reaching $8.6 \%$ in 2020 (Fig. 1a). The number of imported recurrent $P$. ovale cases increased gradually from 2013 to 2018 and then slightly decreased in 2019 and 2020. This trend was mainly associated with the increasing number of imported P. ovale cases from 2013 to 2020. Additionally, the proportion of imported recurrent $P$. ovale cases increased continuously after 2013, reaching $16.2 \%$ in 2020.

Table 1 Demographic characteristics of imported recurrence cases of Plasmodium vivax and P. ovale

\begin{tabular}{|c|c|c|c|c|c|}
\hline \multirow[t]{3}{*}{ Demographic characteristics } & \multicolumn{4}{|c|}{ Imported recurrent cases } & \multirow[t]{3}{*}{$P$ value } \\
\hline & \multicolumn{2}{|l|}{ P. vivax } & \multicolumn{2}{|l|}{ P. ovale } & \\
\hline & Number & $\%$ & Number & $\%$ & \\
\hline Total & 179 & & 128 & & \\
\hline Sex & & & & & 0.3869 \\
\hline Male & 163 & $91.1 \%$ & 120 & $93.8 \%$ & \\
\hline Female & 16 & $8.9 \%$ & 8 & $6.3 \%$ & \\
\hline Age (years) & & & & & $<0.0010$ \\
\hline$<10$ & 0 & $0.0 \%$ & 0 & $0.0 \%$ & \\
\hline $10-19$ & 2 & $1.1 \%$ & 1 & $0.8 \%$ & \\
\hline $20-29$ & 50 & $27.9 \%$ & 21 & $16.4 \%$ & \\
\hline $30-39$ & 51 & $28.5 \%$ & 46 & $35.9 \%$ & \\
\hline $40-49$ & 50 & $27.9 \%$ & 26 & $20.3 \%$ & \\
\hline $50-59$ & 20 & $11.2 \%$ & 32 & $25.0 \%$ & \\
\hline$\geq 60$ & 6 & $3.4 \%$ & 2 & $1.6 \%$ & \\
\hline Occupation* & & & & & $<0.0500$ \\
\hline Migrant workers & 32 & $43.2 \%$ & 42 & $44.7 \%$ & \\
\hline Construction workers & 29 & $38.2 \%$ & 20 & $21.3 \%$ & \\
\hline Other workers ${ }^{\#}$ & 15 & $19.7 \%$ & 32 & $34.0 \%$ & \\
\hline Civil servants & 9 & $8.7 \%$ & 5 & $4.6 \%$ & \\
\hline Businessmen & 5 & $4.9 \%$ & 3 & $2.75 \%$ & \\
\hline Others $^{\S}$ & 13 & $12.6 \%$ & 7 & $6.4 \%$ & \\
\hline Radical curative treatment* & & & & & 0.9001 \\
\hline Yes & 9 & $8.7 \%$ & 9 & $8.3 \%$ & \\
\hline No & 94 & $91.3 \%$ & 100 & $91.7 \%$ & \\
\hline
\end{tabular}

*Data for occupation and radical curative treatment only included data from 2017 to 2020, as they were not recorded in 2013-2016. ${ }^{\#}$ Other workers included carpenters, electricians and miners. ${ }^{\S}$ Others included travelers, students and migrants 


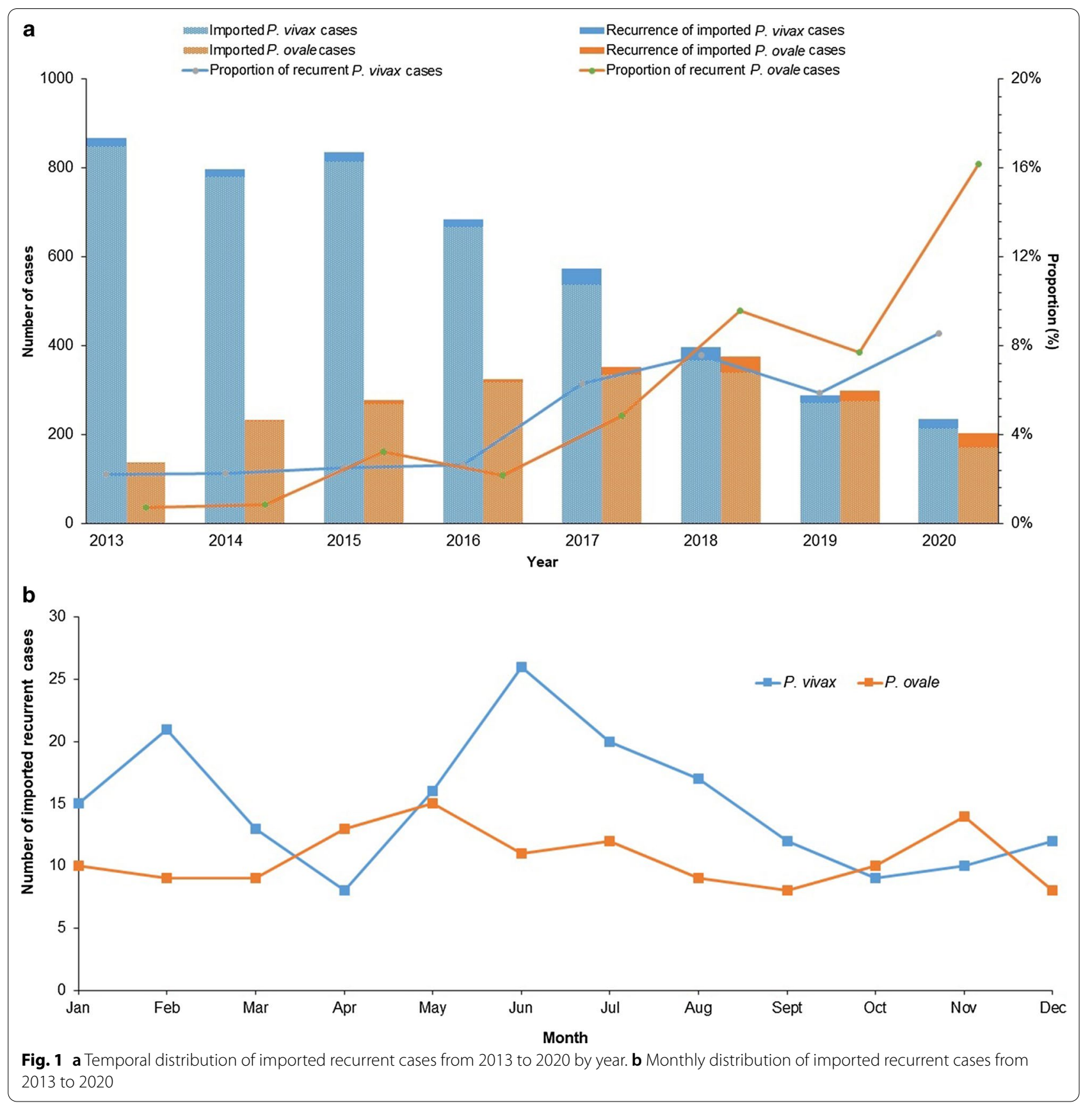

The monthly distribution of imported recurrent cases exhibited seasonal changes from 2013 to 2020 (Fig. 1b). Recurrent $P$. vivax cases showed two peaks in February and June, accounting for $11.7 \%$ and $14.5 \%$ of the total number of imported recurrent cases of $P$. vivax, respectively. In contrast, the monthly distribution of recurrent cases of $P$. ovale over the whole year was relatively stable, with slight increases in May and November.

\section{Spatial distributions of imported recurrent cases}

The majority of recurrent $P$. vivax cases were imported from Southeast Asia (53.6\%, 96/179), followed by East Africa (17.2\%, 29/179), South Asia (12.4\%, 21/179), West Africa (8.9\%, 15/179), and Central Africa (7.1\%, 12/179). Among these cases, the source countries were Myanmar, Ethiopia, and Pakistan, accounting for $43.0 \%, 12.9 \%$, and $11.2 \%$, respectively (Fig. 2 and Additional file 1). In 


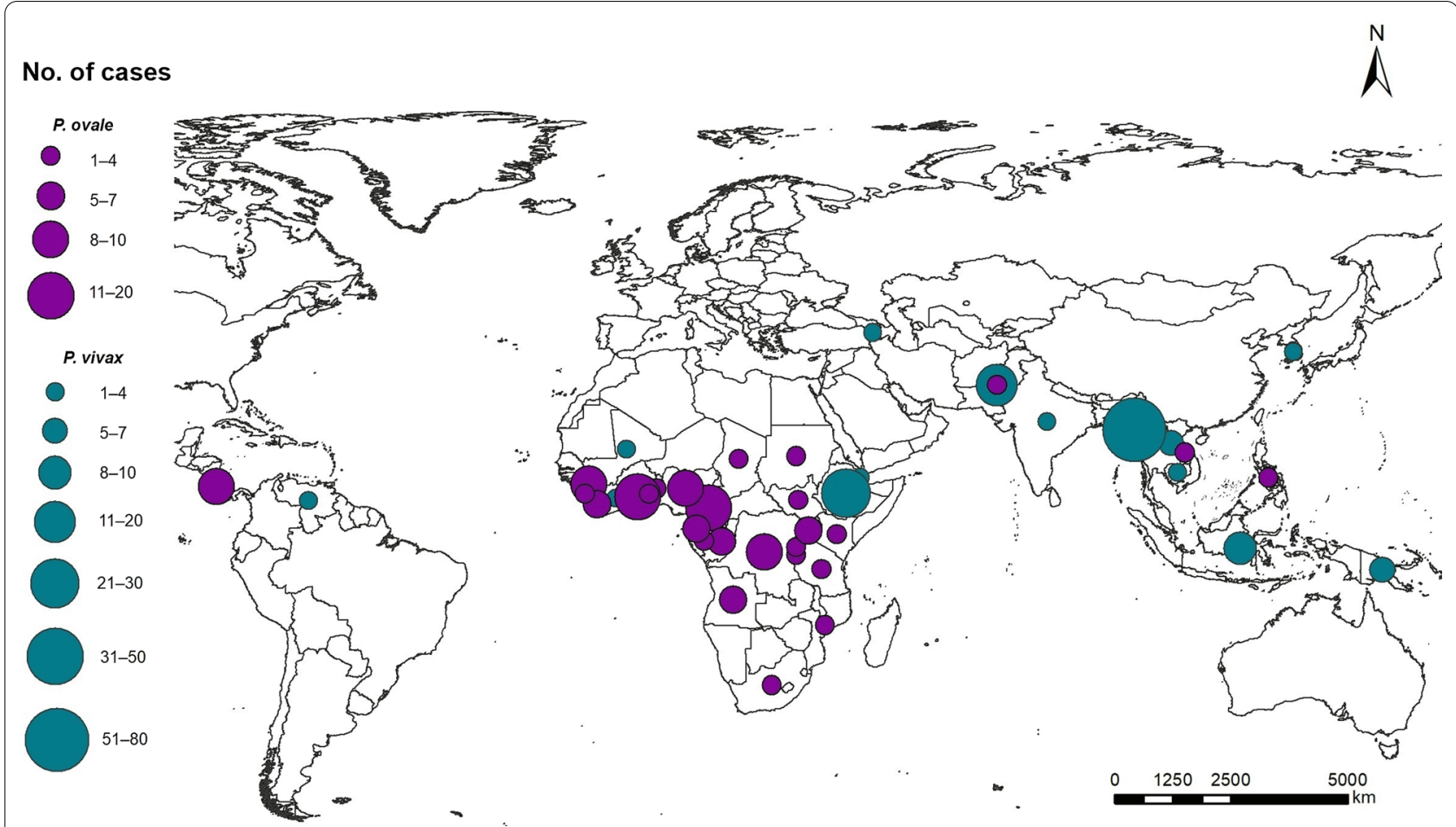

Fig. 2 Source countries of imported recurrent Plasmodium vivax and P. ovale cases from 2013 to 2020. The world map is downloaded from https:// www.naturalearthdata.com/

addition, most cases were reported in Yunnan Province (41.3\%), followed by Sichuan (10.6\%) and Henan (6.7\%) provinces (Fig. 3 and Additional file 2).

The majority of recurrent $P$. ovale cases were imported from Central Africa $(40.0 \%, 51 / 128)$ and West Africa (39.1\%, 50/128), while small proportions were from East Africa, South Africa, Asia, and North Africa, accounting for $8.9 \%(11 / 128), 8.1 \%(10 / 128), 3.2 \%$ (4/128), and $1.6 \%$ (2/128), respectively. The main source countries included Cameroon, Ghana, and Nigeria, which accounted for $13.3 \%, 10.9 \%$, and $7.8 \%$, respectively (Fig. 2 and Additional file 1). Imported recurrent cases of $P$. ovale were primarily reported in southern China, which has a large immigrant population. The main provinces included Guangxi, Guangdong, Henan and Zhejiang, accounting for $18.0 \%, 12.5 \%, 9.4 \%$ and 9.4\%, respectively (Fig. 3 and Additional file 3).

\section{Interval from previous infection to recurrence in patients with imported recurrent cases}

The interval from previous infection to recurrence was recorded in only 293 patients, including 169 patients with $P$. vivax infection and 124 patients with $P$. ovale infection. The interval in P. ovale infection patients [308 days, interquartile range (IQR): 162-510] was longer than that in $P$. vivax infection patients (183 days, IQR: $85-305$ )
(Fig. 4a). The $P$. vivax intervals among regions were significantly different $(P=0.0016)$, with the shortest in East Africa (116 days, IQR: 78-364), followed by West Africa (139 days, IQR: 97-248) and Southeast Asia (144 days, IQR: 75-268) (Fig. 4b). The recurrent $P$. ovale interval was shortest in South Africa (183 days, IQR: 81-521), followed by East Africa (221 days, IQR: 145-344), Central Africa (277 days, IQR: 159-475), West Africa (360 days, IQR: 218-583), and Asia (522 days, IQR: 397-679) (Fig. 4c). The median interval in the total patients with recurrent $P$. ovale infection from Africa was 308 days (IQR: 168-476). However, the interval was not significantly different among the different regions $(P=0.2373)$, and no significant difference was observed between Africa and Asia $(P=0.4053)$.

\section{Discussion}

P. vivax poses a significant challenge to the elimination of malaria due to its ability to cause relapsed infection by the reactivation of dormant liver parasites called hypnozoites [16]. While China has achieved elimination, patients with $P$. vivax and $P$. ovale infections have the potential to relapse weeks, months, or years after primary infection and may not present symptoms when they arrive in China [17]. Consequently, patients and physicians are less likely to link a febrile illness with travel, 


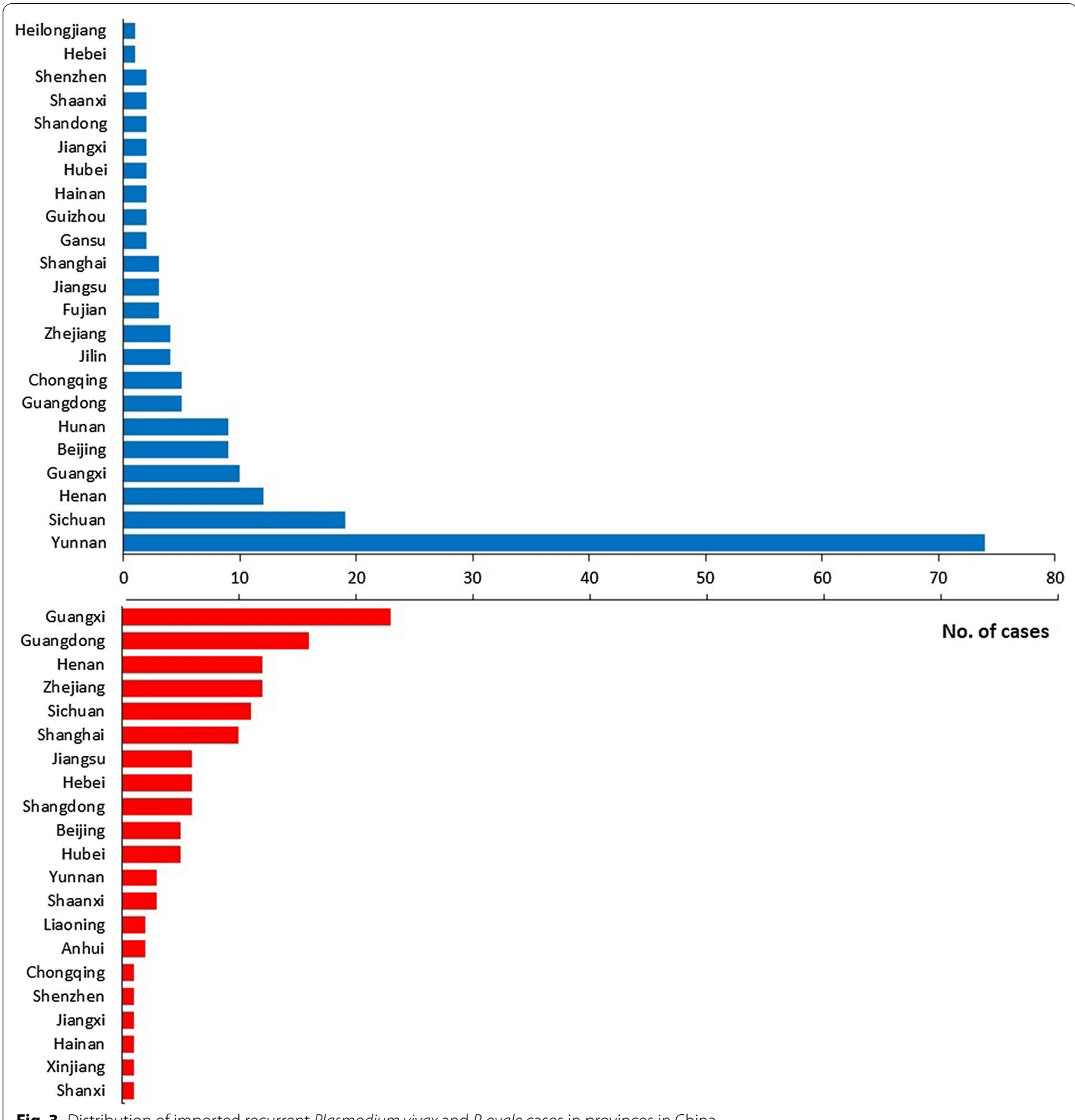

Fig. 3 Distribution of imported recurrent Plasmodium vivax and P. ovale cases in provinces in China

resulting in delayed or missed diagnosis [18]. Therefore, it is of great significance to analyse the epidemiological characteristics of imported recurrent cases to provide evidence to guide the development of appropriate public health intervention strategies in the post-elimination settings.

In the present study, all imported recurrent $P$. vivax and $P$. ovale cases from 2013 to 2020 were included. The majority of imported recurrent cases occurred in males (P. vivax $91.1 \%$, P. ovale $93.8 \%)$ and migrant workers $(P$. vivax $43.2 \%$, P. ovale $44.7 \%$ ) (Table 1 ), which was consistent with the distribution of total imported cases by sex (males, 94.9\%) and occupation (migrant workers, 68.3\%) [12]. This result may have been due to the increasing number of Chinese migrant workers that travel abroad, especially to malaria-endemic countries or regions. The 


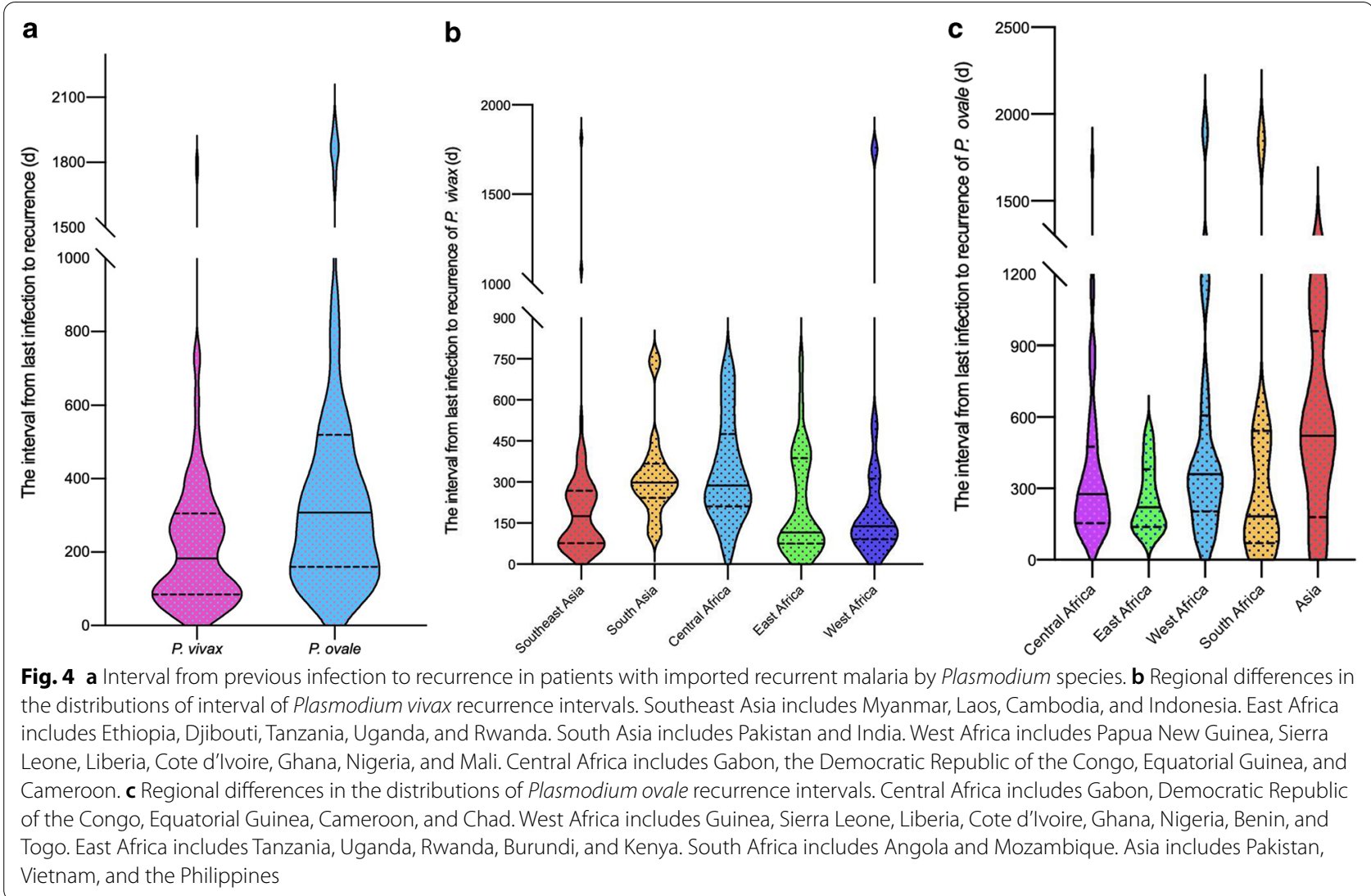

groups most affected by $P$. vivax and $P$. ovale were individuals aged $30-39$ years, indicating that young adults may have the highest risk of recurrent infection. One study identified that the median age of patients with imported recurrent cases in the United Kingdom from 1987 to 2015 was 32 years (IQR: 22-45) [19]. Another study found that the highest proportion of local recurrent $P$. vivax cases in Nepal occurred in individuals aged 21-30 years (35.7\%), followed by $11-20$ years (27\%) [20]. The age group with the highest risk of recurrent malaria was similar to the age group comprising the largest proportion of the migrant population in China.

Compared with the number of recurrent cases in China before 2013 [21], the number of imported recurrent $P$. vivax and $P$. ovale cases has increased since 2013 (Fig. 1a). This rising trend might be caused by several reasons. First is the implementation of a new web-based case reporting system, PDIRMS, which has recorded information about recurrent cases since 2013. Secondly, the status of radical treatment strategies, G6PD deficiency rate, treatment adherence, and drug resistance in the infection source countries primarily associated with imported malaria cases were other key causes.

Radical treatment strategies for $P$. vivax and $P$. ovale were not the same in different countries, and even not large-scale adoption in some of the infection source countries. According to the antimalarial drug policy in China [22], recurrent $P$. vivax and $P$. ovale cases were treated with a standard regimen of 3-day chloroquine (CQ) and concurrent 8-day PQ (22.5 mg/day) for radical cure, while the first-line treatment for $P$. vivax malaria in most countries, including Myanmar, the main source country of recurrent $P$. vivax infections, is 3-day CQ and 14-day PQ (3.5 mg/kg total dose) [23, 24]. Most recurrent $P$. ovale cases were imported from Central Africa (Cameroon) and West Africa (Ghana, Nigeria) (Fig. 2). However, PQ is not used for radical cure of $P$. vivax and P. ovale malaria in Cameroon, Ghana, and Nigeria [25]. This may explain the increasing number of imported recurrent cases from these countries, which have a low rate of radical cure (P. vivax $8.7 \%$, P. ovale $8.3 \%$ ).

G6PD deficiency greatly hinders the widespread use of PQ, because PQ could cause haemolysis in people with G6PD deficiency [8]. The prevalence of G6PD deficiency was relatively high in the major source countries [26], including Myanmar (3-7\%), Cameroon (10-13\%), Ghana (17-20\%), and Nigeria (13-17\%). Moreover, a high prevalence of G6PD deficiency (29.6\%) was detected in the Kachin ethnic group (Jingpo) along the China-Myanmar border [27]. Thus, radical cure cannot be widely adopted 
and patients are reluctant to take $\mathrm{PQ}$, especially if they cannot be tested for G6PD deficiency [28]. In accordance with the malaria elimination programme in Myanmar, $P$. vivax patients were given a weekly dose of $0.75 \mathrm{mg} / \mathrm{kg}$ PQ for 8 weeks by village health volunteers or integrated community malaria volunteers in the community; this regimen may be widely used and safer in those without G6PD deficiency testing [29].

In addition, PQ is generally prescribed as a 14-day course for radical treatment, which hampers treatment adherence because most patients discontinue their medication as soon as their symptoms disappear. In China, an 8-day course of PQ was used to improve treatment adherence [22], and one study also showed that a short 7- to 9-day short course of PQ with CQ was equally as effective as the 14-day regimen in preventing relapse [30].

Drug resistance causes recrudescence (blood-stage treatment failure), another kind of recurrence. CQ resistance was first reported in Papua New Guinea in 1989 [31]. It then spread to Indonesia, Brazil, Myanmar, India, Cambodia, and Ethiopia [32-37]. Although imported recurrent $P$. vivax malaria in China was mainly imported from Myanmar, $P$. vivax was mostly sensitive to $C Q$, with treatment failure rates of less than 5\% along the ChinaMyanmar border [38] and 2.6\% in northeast Myanmar [39]. Although the $C Q / P Q$ treatment failure rate in patients with $P$. vivax infection has been relatively low near the China-Myanmar border, CQ-resistant P. vivax has emerged in Greater Mekong subregion (GMS) and some countries have reported a highly resistant phenotype. In this study, most recurrent $P$. ovale cases were primarily imported from Cameroon, Ghana, and Nigeria, while there have been few reports about the $C Q$ resistance profile of $P$. vivax and $P$. ovale in Cameroon, Ghana, and Nigeria, as $P$. falciparum is the predominant species in such areas. In addition, artemisinin combination therapies (ACTs) were recommended by WHO as the first- and second-line treatment for uncomplicated P. falciparum malaria as well as for chloroquine-resistant $P$. vivax malaria, which have already been used to treat infections by $P$. falciparum and mixed species resistant to CQ in Cameroon [40].

This study found that the number of imported recurrent cases of $P$. vivax displayed well-defined seasonality, with two peaks in February and June, respectively (Fig. 1b). The number of recurrent $P$, ovale cases increased slightly in May and November; these increases were related to holidays associated with family visits, increasing the number of imported cases. According to the analysis of imported malaria cases in China, imported malaria cases associated with the end of the work season or studying abroad were concentrated in May-July, while malaria cases associated with the Spring Festival/summer vacation or a return home to visit relatives were mostly distributed in January and May [41].

The intervals between infections in those with imported recurrent $P$. vivax cases among different continents were significantly different and may have been influenced by geographic and environmental factors [42-44]. Previous studies found that temperate and subtropical strains of $P$. vivax often exhibited either a long incubation or latent period of around eight to ten months, while tropical strains were characterized by short incubation times and short latency (approximately three to six weeks). In addition, Recurrence frequency may result from evolved responses to average transmission season duration and/ or vector suitability periods. The median time from primary infection to relapse in patients with $P$. vivax malaria was different among nine different regions, i.e., 107 days and 41 days in sub-Saharan Africa and Southeast Asia, respectively [45]. In the present study, the median infection interval in Southeast Asia was 144 days, which was relatively long, possibly because the data analysed herein comprised the interval from previous infection to recurrence instead of from primary infection to relapse, and the data were collected from patient' self-reports rather than a cohort study.

Some limitations were noted in this study. First, genotyping methods have not been able to precisely differentiate relapse from new infection or recrudescence. None of the imported recurrent cases in this study were stratified into relapse, recrudescence, or reinfection subgroups. Secondly, information for tracing the infection source countries of imported malaria cases is not available. Thirdly, data on the interval from previous infection to recurrence were collected based on patient self-reports rather than a cohort study. In addition, $P$. ovale curtisis and $P$. ovale wallikeri, two genetically distinct subspecies of $P$. ovale, were not differentiated in the study.

\section{Conclusions}

Large numbers of imported recurrent cases have been a major challenge in achieving and maintaining malaria elimination in China. This study provides data to guide the development of appropriate public health intervention strategies for imported recurrent $P$. vivax and $P$. ovale cases. A surveillance system based on the "1-3-7" approach should be fully operational with integrated drug efficiency surveillance to identify recurrent cases in the post-elimination phase. In addition, the radical cure rate and the capability of G6PD deficiency testing in patients with imported cases should be strengthened. Targeted intervention strategies combined with powerful surveillance could sustain malaria elimination and prevent re-establishment in China. 


\section{Supplementary Information}

The online version contains supplementary material available at https://doi. org/10.1186/s40249-021-00896-3.

Additional file 1. Distribution of imported recurrent Plasmodium vivax cases at the provincial level in China.

Additional file 2. Distribution of imported recurrent Plasmodium ovale cases at the provincial level in China.

Additional file 3. Numbers of imported recurrent Plasmodium vivax and $P$. ovale cases from different source countries from 2013 to 2020.

\section{Acknowledgements}

We thank the staff for their effort and assistance in data collection at different levels of the case reporting system in China.

\section{Authors' contributions}

FH conceived and designed the study; LZ and YC contributed to data collection; ZX contributed to the review and editing of the manuscript; $Y C$ and $\mathrm{JH}$ carried out the data analysis; $\mathrm{YC}$ and FH drafted the manuscript. All authors read and approved the final manuscript.

\section{Funding}

This work was supported by the National Important Scientific \& Technological Project (2018ZX10101002-002), the Fifth Round of Three-Year Public Health Action Plan of Shanghai (No. GWV-10.1-XK13), the Natural Science Foundation of Shanghai (No. 18ZR1443400) and the National Malaria Elimination Program of China.

\section{Availability of data and materials}

The datasets used and/or analysed during the current study are available from the corresponding author upon reasonable request.

\section{Declarations}

Ethics approval and consent to participate

Not applicable.

\section{Consent for publication}

Not applicable.

\section{Competing interests}

The authors have no competing interests to disclose.

\section{Author details}

${ }^{1}$ National Institute of Parasitic Diseases, Chinese Center for Disease Control and Prevention (Chinese Center for Tropical Diseases Research), Shanghai 200025, China. ${ }^{2} \mathrm{NHC}$ Key Laboratory of Parasite and Vector Biology, Shanghai 200025, China. ${ }^{3} \mathrm{WHO}$ Collaborating Centre for Tropical Diseases, Shanghai 200025, China. ${ }^{4}$ National Center for International Research on Tropical Diseases, Shanghai 200025, China.

Received: 7 July 2021 Accepted: 12 Auqust 2021

Published online: 23 August 2021

\section{References}

1. WHO. World malaria report 2020. Geneva: World Health Organization; 2020.

2. Gething PW, Elyazar IR, Moyes CL, Smith DL, Battle KE, Guerra CA, et al. A long neglected world malaria map: Plasmodium vivax endemicity in 2010. PLoS Negl Trop Dis. 2012;6: e1814.

3. WHO. World malaria report 2015. Geneva: World Health Organization; 2015.

4. Howes RE, Battle KE, Mendis KN, Smith DL, Cibulskis RE, Baird JK, et al. Global epidemiology of Plasmodium vivax. Am J Trop Med Hyg. 2016:95:15-34.
5. Kerkhof K, Canier L, Kim S, Heng S, Sochantha T, Sovannaroth S, et al. Implementation and application of a multiplex assay to detect malariaspecific antibodies: a promising tool for assessing malaria transmission in Southeast Asian pre-elimination areas. Malar J. 2015;14:338.

6. Dayananda KK, Achur RN, Gowda DC. Epidemiology, drug resistance, and pathophysiology of Plasmodium vivax malaria. J Vector Borne Dis. 2018;55:1-8.

7. Taylor AR, Watson JA, Chu CS, Puaprasert K, Duanguppama J, Day NPJ, et al. Resolving the cause of recurrent Plasmodium vivax malaria probabilistically. Nat Commun. 2019;10:5595.

8. Baird JK, Valecha N, Duparc S, White NJ, Price RN. Diagnosis and treatment of Plasmodium vivax Malaria. Am J Trop Med Hyg. 2016;95:35-51.

9. Zhu DS, Yang WZ, Wang JJ. Progress on the long incubation period of Plasmodium vivax. Zhonghua Liu Xing Bing Xue Za Zhi. 2008;29:81-4 (In Chinese).

10. Feng $X$, Xia ZG, Feng J, Zhang L, Yan H, Tang L, et al. The contributions and achievements on malaria control and forthcoming elimination in China over the past 70 years by NIPD-CTDR. Adv Parasitol. 2020;110:63-105.

11. Lai S, Sun J, Ruktanonchai NW, Zhou S, Yu J, Routledge I, et al. Changing epidemiology and challenges of malaria in China towards elimination. Malar J. 2019;18:107.

12. Feng J, Zhou SS. From control to elimination: the historical retrospect of malaria control and prevention in China. Chin J Parasit Dis. 2019;37:50513 (In Chinese).

13. Lin JT, Saunders DL, Meshnick SR. The role of submicroscopic parasitemia in malaria transmission: what is the evidence? Trends Parasitol. 2014;30:183-90.

14. malERA Consultative Group on Diagnoses and Diagnostics. A research agenda for malaria eradication: drugs. PLoS Med. 2011;8: e1000402.

15. China NHC. Diagnosis of malaria (WS259-2015). Beijing: National Health Commission of the People's Republic China; 2016.(In Chinese).

16. Cowell AN, Valdivia HO, Bishop DK, Winzeler EA. Exploration of Plasmodium vivax transmission dynamics and recurrent infections in the Peruvian Amazon using whole genome sequencing. Genome Med. 2018;10:52.

17. White NJ. Determinants of relapse periodicity in Plasmodium vivax malaria. Malar J. 2011;10:297.

18. Morgan GS, Chiodini P, Evans M. Relapsing malaria: two cases of malaria presenting 8 months after return from Africa despite adherence to antimalarial chemoprophylaxis. Br J Gen Pract. 2012;62:555-6.

19. Nabarro LEB, Nolder D, Broderick C, Nadjm B, Smith V, Blaze M, et al. Geographical and temporal trends and seasonal relapse in Plasmodium ovale spp. and Plasmodium malariae infections imported to the UK between 1987 and 2015. BMC Med. 2018;16:218.

20. Manandhar S, Bhusal CL, Ghimire U, Singh SP, Karmacharya DB, Dixit SM. A study on relapse/re-infection rate of Plasmodium vivax malaria and identification of the predominant genotypes of $P$. vivax in two endemic districts of Nepal. Malar J. 2013;12:324.

21. Xia ZG, Feng J, Zhou SS. Malaria situation in the People's Republic of China in 2012. Zhongguo Ji Sheng Chong Xue Yu Ji Sheng Chong Bing Za Zhi. 2013:31:413-8 (In Chinese).

22. China NHC. Technical regulations for application of antimalarials. Beijing: National Health Commission of the People's Republic China; 2015. (In Chinese).

23. Chu CS, Phyo AP, Lwin KM, Win HH, San T, Aung AA, et al. Comparison of the cumulative efficacy and safety of chloroquine, artesunate, and chloroquine-primaquine in Plasmodium vivax malaria. Clin Infect Dis. 2018;67:1543-9.

24. Hewitt S, Delacollette C, Chavez I. Malaria situation in the Greater Mekong Subregion. Southeast Asian J Trop Med Public Health. 2013;44(Suppl 1):46-72; discussion 306-7.

25. Badens C, Martinez di Montemuros F, Thuret I, Michel G, Mattei JF, Cappellini MD, et al. Molecular basis of haemoglobinopathies and G6PD deficiency in the Comorian population. Hematol J. 2000;1:264-8.

26. Howes RE, Piel FB, Patil AP, Nyangiri OA, Gething PW, Dewi M, et al. G6PD deficiency prevalence and estimates of affected populations in malaria endemic countries: a geostatistical model-based map. PLoS Med. 2012;9: e1001339.

27. Li Q, Yang F, Liu R, Luo L, Yang Y, Zhang L, et al. Prevalence and molecular characterization of glucose-6-phosphate dehydrogenase deficiency at the China-Myanmar border. PLoS One. 2015;10:e0134593. 
28. Zhang L, Yang Y, Liu R, Li Q, Yang F, Ma L, et al. A multiplex method for detection of glucose-6-phosphate dehydrogenase (G6PD) gene mutations. Int J Lab Hematol. 2015;37:739-45.

29. Recht J, Ashley EA, White NJ. Use of primaquine and glucose-6-phosphate dehydrogenase deficiency testing: divergent policies and practices in malaria endemic countries. PLoS Negl Trop Dis. 2018;12: e0006230.

30. Daher A, Silva J, Stevens A, Marchesini P, Fontes CJ, Ter Kuile FO, et al. Evaluation of Plasmodium vivax malaria recurrence in Brazil. Malar J. 2019:18:18.

31. Whitby M, Wood G, Veenendaal JR, Rieckmann K. Chloroquine-resistant Plasmodium vivax. Lancet. 1989;2:1395.

32. Baird JK, Basri H, Purnomo, Bangs MJ, Subianto B, Patchen LC, et al. Resistance to chloroquine by Plasmodium vivax in Irian Jaya, Indonesia. Am J Trop Med Hyg. 1991;44:547-52.

33. Garavelli PL, Corti E. Chloroquine resistance in Plasmodium vivax: the first case in Brazil. Trans R Soc Trop Med Hyg. 1992;86:128.

34. Myat Phone K, Myint O, Myint L, Thaw Z, Kyin Hla A, Nwe NY. Emergence of chloroquine-resistant Plasmodium vivax in Myanmar (Burma). Trans $R$ Soc Trop Med Hyg. 1993;87:687.

35. Soto J, Toledo J, Gutierrez P, Luzz M, Llinas N, Cedeno N, et al. Plasmodium vivax clinically resistant to chloroquine in Colombia. Am J Trop Med Hyg. 2001;65:90-3.

36. Teka H, Petros B, Yamuah L, Tesfaye G, Elhassan I, Muchohi S, et al. Chloroquine-resistant Plasmodium vivax malaria in Debre Zeit. Ethiopia Malar J. 2008;7:220.

37. Van den Abbeele K, Van den Enden E, Van den Ende J. Combined chloroquine and primaquine resistant Plasmodium vivax malaria in a patient returning from India. Ann Soc Belg Med Trop. 1995;75:73-4.
38. Liu H, Yang HL, Tang LH, Li XL, Huang F, Wang JZ, et al. Monitoring Plasmodium vivax chloroquine sensitivity along China-Myanmar border of Yunnan Province, China during 2008-2013. Malar J. 2014;13:364.

39. Xu S, Zeng W, Ngassa Mbenda HG, Liu H, Chen X, Xiang Z, et al. Efficacy of directly-observed chloroquine-primaquine treatment for uncomplicated acute Plasmodium vivax malaria in northeast Myanmar: a prospective open-label efficacy trial. Travel Med Infect Dis. 2020;36: 101499.

40. Antonio-Nkondjio C, Ndo C, Njiokou F, Bigoga JD, Awono-Ambene P, Etang J, et al. Review of malaria situation in Cameroon: technical viewpoint on challenges and prospects for disease elimination. Parasit Vectors. 2019;12:501.

41. Garrido-Cardenas JA, Cebrián-Carmona J, González-Cerón L, ManzanoAgugliaro F, Mesa-Valle C. Analysis of global research on malaria and Plasmodium vivax. Int J Environ Res Public Health. 2019;16(11):1928.

42. Mueller I, Galinski MR, Baird JK, Carlton JM, Kochar DK, Alonso PL, et al. Key gaps in the knowledge of Plasmodium vivax, a neglected human malaria parasite. Lancet Infect Dis. 2009;9:555-66.

43. Douglas NM, Nosten F, Ashley EA, Phaiphun L, van Vugt M, Singhasivanon $P$, et al. Plasmodium vivax recurrence following falciparum and mixed species malaria: risk factors and effect of antimalarial kinetics. Clin Infect Dis. 2011;52:612-20.

44. Shanks GD, White NJ. The activation of vivax malaria hypnozoites by infectious diseases. Lancet Infect Dis. 2013;13:900-6.

45. Battle KE, Karhunen MS, Bhatt S, Gething PW, Howes RE, Golding N, et al. Geographical variation in Plasmodium vivax relapse. Malar J. 2014;13:144.
Ready to submit your research? Choose BMC and benefit from:

- fast, convenient online submission

- thorough peer review by experienced researchers in your field

- rapid publication on acceptance

- support for research data, including large and complex data types

- gold Open Access which fosters wider collaboration and increased citations

- maximum visibility for your research: over 100M website views per year

At BMC, research is always in progress.

Learn more biomedcentral.com/submissions 